\title{
GALACTIC DYNAMO THEORY CONFRONTED WITH OBSERVATIONS
}

\author{
YU.S. KRASHENINNIKOVA \\ Space Research Institute, Moscow 117810, USSR \\ D.D. SOKOLOFF \\ Physics Department, Moscow University, Moscow 119899, USSR \\ A.A. RUZMAIKIN and A. SHUKUROV \\ IZMIRAN, Troitsk, Moscow Region 142092, USSR
}

\begin{abstract}
Kinematic models of galactic dynamo in axisymmetric disk show remarkable agreement with observations. We argue that nonlinear effects are relatively weak in galactic dynamos and consider the properties of linear dynamo models inherited by global magnetic configurations in spiral galaxies as well as nonlinear distortions of the linear solutions.
\end{abstract}

\section{Introduction}

The galactic dynamo theory is remarkably advantageous for applications since even its simplest kinematic version yields the results which can be directly compared with observations of spiral galaxies. On the other hand, it is clear that nonlinear effects can play some role in galactic dynamos and one should sensibly select those parameters of linear dynamo solutions which can be inherited by observed global magnetic configurations. (Nonlinear dynamo effects arise owing to influence of the generated magnetic field on fluid motions).

Basic facts used in this paper and detailed references can be found in recent reviews of Sofue et al. (1986), Beck (1986), Ruzmaikin et al. (1988a,b) and F. Krause (1989).

\section{Asymptotic Models of Galactic Dynamo}

Up to now, two approaches to the galactic dynamo theory have proved to be most fruitful. The first one considers generation of the large-scale magnetic field in a thin disk and applies to outer parts of galaxies beyond a few kiloparsecs from their centers. To the lowest approximation, the generation is considered locally, at a fixed radius, and global solutions are constructed on the basis of local ones.

Solutions of the dynamo equation in a thin disk depend most strongly on $z$-coordinate measured across the disk. However, radial variations of the generation strength and disk thickness introduce a weak parametric dependence of the local solutions $\mathbf{b}$ on the radius, $r$. In can be shown that the radial scale of magnetic structures generated by the thin-disk dynamo is somewhat smaller than the disk radius and has the order of magnitude $L \simeq\left(h_{0} r_{0}\right)^{1 / 2}$ with $r_{0}$ the radius and $h_{0}$ the half-thickness of the disk. Thus, asymptotic distribution of the large-scale magnetic field in a thin disk is characterized by three distinct scales and has the form 
$\mathbf{B}=Q(r / L) \mathbf{b}\left(z / h_{0} ; r / r_{0}\right) \exp (i \Gamma t+i m \varphi)$, where $\Gamma$ is the global growth rate and $m$ is the azimuthal wave number $(m=0$ and $m=1$ correspond to axisymmetric and bisymmetric structures, respectively). After introduction of this ansatz the dynamo equation splits into relatively simple equations for $\mathbf{b}$ and $Q$ which can be solved with account for observed parameters of galaxies.

Note that for $r_{0}=10 \mathrm{kpc}$ and $h_{0}=0.4 \mathrm{kpc}$ we have $L \simeq 2 \mathrm{kpc}$ which is of the same order as the radial width of the magnetic ring in M31. This agreement is associated with relative weakness of nonlinear effects in this galaxy. Nonlinear effects result in growth of the radial scale of magnetic field distribution. Indeed, the field growth saturates first near the maximum of the linear distribution but at the wings of the eigenfunction the growth proceeds further for some period. Thus, the radial scale of the field distribution grows at the rate $u \simeq(\gamma \beta)^{1 / 2}$, where $\gamma \simeq$ $\left(5 \times 10^{8} \mathrm{yr}\right)^{-1}$ is the local growth rate of the field and $\beta \simeq 10^{26} \mathrm{~cm}^{2} \mathrm{~s}^{-1}$ is the turbulent magnetic diffusivity. In $T=10^{10}$ yr the radial width of the field distribution can become wider by $\Delta L \simeq 2 u T \simeq 7 \mathrm{kpc}$ and reach the value of the order of $L+\Delta L \simeq$ $10 \mathrm{kpc}$. This value coincides with radial widths of distributions of large-scale magnetic fields in such galaxies as M51 or M81.

In those galaxies where nonlinear effects are relatively weak and the observed field is close to a combination of several linear eigenfunctions, the axisymmetric magnetic field can exhibit reversals along the radius at the scale $L \simeq 2 \mathrm{kpc}$ while the total width of the distribution is about $10 \mathrm{kpc}$ (Ruzmaikin et al., 1985). Probably, such situation occurs in the Milky Way. Thus, alternating large-scale magnetic fields in neighboring spiral arms in the Milky Way hardly can be interpreted as unambiguous evidence of global bisymmetric structure.

An alternative asymptotic solution, applicable to the central regions of galaxies, employs the fact that normally the generation is particularly strong near galactic centers. This solution can be applied to such galaxies as M33, NGC 6946 and IC 342 (Starchenko and Shukurov, 1989). As follows from these asymptotics, in the very centers of galaxies the field can have dipole symmetry and large vertical component.

\section{Imprints of Kinematic Dynamo Modes in Observed Magnetic Structures}

When magnetic field cannot be excited in certain part of galactic disk at the linear stage of galactic dynamo, one can hardly expect to find there a more or less strong steady-state magnetic field. Indeed, as can be seen from expression for $u$ above, the large-scale magnetic field cannot spread to the region where $\gamma<0$. This simple fact explains the existence of a peculiar magnetic annulus in M31: due to a specific form of the rotation curve, $\gamma<0$ for $2<r<7 \mathrm{kpc}$ and magnetic field distribution can spread only outwards (a similar magnetic ring probably exists in the Milky Way).

Another important property of the large-scale magnetic field, which is formed at the linear stage, is the trailing spiral shape (rather than circular) of magnetic lines (which close outside the disk, probably in the corona). Observations confirm this theoretical assessment. Moreover, observed pitch angles of spiral magnetic lines reasonably agree with predictions of the dynamo theory. This agreement has the following rather deep reason. Nonlinear effects in galactic dynamos can be considered as weak perturbations. Then the perturbation theory predicts that, to the leading order, nonlinearity reduces the growth rate of the field but does not affect the ratio $B_{r} / B_{\varphi}$ which determines the pitch angle of magnetic lines.

The most urgent problem of both theory and observations now seems to be verification and explanation of possible dominance of bisymmetric magnetic mode $m=1$ 
in at least several spiral galaxies. Observations indicate the dominance of bisymmetric structure in M81 while the axisymmetric mode $m=0$ dominates in M31 and IC 342 (see Beck, 1989).

For those galaxies where observations reveal only the $m=0$ mode, the linear theory also asserts that this is the only excited mode. For those galaxies where presence of the mode $m=1$ is admissible, the theory also indicates the possibility of excitation of this mode.

Note that the dynamo theory developed for axisymmetric disks implies that, along with the $m=1$ dynamo mode, the $m=0$ mode is always generated at greater growth rate. It would seem natural that dominance of the $m=1$ mode can be explained by deviations of galactic disks from exact axial symmetry. However, it has been shown that weak perturbations from spiral arms do not facilitate generation of the $m=1$ mode since spiral patterns have higher azimuthal wave number, $m=2$. Perturbations associated with global asymmetry of galactic disks are too weak to make the $m=1$ mode dominant and can explain only deviations from axial symmetry in basically axisymmetric configurations exemplified by M31 (Ruzmaikin et al., 1989). More promising is warping of galactic disk which possibly can lead to dominance of the $m=1$ dynamo mode. There also exists a wide selection of effects, which still have not been analyzed in detail, but which are potentially capable of making the $m=1$ mode dominant at the linear stage. Here we mention the anisotropy of the mean helicity of interstellar turbulence, radial flows of interstellar gas, the role of boundary conditions on disk surface, etc.

The galactic dynamo theory predicts very definitely that in the main parts of all galactic disks only the large-scale fields with even symmetry with respect to galactic equator can be generated. Available observational possibilities restrict relevant observations to the solar vicinity of the Milky Way where the theory is confirmed. Recent ideas of $\mathrm{M}$. Krause (1989) possibly can lead to verification of this prediction in external galaxies as well.

\section{How Strong Are Nonlinear Effects in Galactic Dynamos?}

In a certain sense, the mean-field galactic dynamos are very weak (Krasheninnikova et al., 1989). The large-scale magnetic field grows so slowly that, for example, in the Milky Way and M31 only three characteristic growth times span the galactic lifetime. Thus, the seed magnetic field produced by stellar outflows (Ruzmaikin et al., $1988 \mathrm{~b}$ ) just has the time to grow up to the observed strength. In such galaxies as M51 and M81 the generation proceeds faster by the order of magnitude. For comparison, note that a few million growth times of solar magnetic field have passed during its lifetime.

We do not have conclusive evidence that the large--scale magnetic fields in the solar vicinity of the Milky Way and in the magnetic ring of M31 have reached the steady state. Concerning, e.g., M51 or M81, we are sure that in these galaxies the field growth has already saturated at the level determined by the balance of the Lorentz and Coriolis forces, $B_{r} B_{\varphi} /(4 \pi h) \simeq \rho \imath \Omega$, where $\rho$ is the gas density, $v$ is the r.m.s. turbulent velocity, and $\Omega$ is the angular velocity of galactic rotation. The dynamo theory establishes the relation between the field components in terms of observable hydrodynamic parameters in galaxies. For the Milky Way, this estimate of the steady-state field strength yields $B_{\varphi} \simeq 7 \mu \mathrm{G}$; it is not clear whether the difference of this estimate from the observed strength, $2-3 \mu \mathrm{G}$, is due to inaccuracy of 
the crude estimate or to non-stationarity of the observed field. In any rate, nonlinearity can be considered weak in galactic dynamos.

\section{References}

Beck, R. (1986) IEEE Trans. Plasma Sci. PS-14, 740.

Beck, R. (1989) Geophys. Astrophys. Fluid Dyn. (in press).

Krasheninnikova, Yu., Ruzmaikin, A., Sokoloff, D. and Shukurov, A. (1989) Geophys. Astrophys. Fluid Dyn. (in press).

Krause, F. (ed.) (1989) Proc. Potsdam Workshop on Magnetic Fields of Galaxies, Geophys. Astrophys. Fluid Dyn. (in press).

Krause, M. (1989) Geophys. Astrophys. Fluid Dyn. (in press).

Ruzmaikin, A., Sokoloff, D. and Shukurov, A. (1985) Astron. Astrophys. 148, 335.

Ruzmaikin, A.A., Shukurov, A.M. and Sokoloff, D.D. (1988a) Magnetic Fields of Galaxies, Kluwer Academic Publishers, Dordrecht.

Ruzmaikin, A., Sokoloff, D. and Shukurov, A. (1988b) Nature 336, 341.

Ruzmaikin, A., Sokoloff, D., Shukurov, A. and Beck, R. (1989) Astron. Astrophys. (in press).

Sofue, Y., Fujimoto, M. and Wielebinski, R. (1986) Ann. Rev. Astron. Astrophys. $24,459$.

Starchenko, S.V. and Shukurov, A. (1989) Astron. Astrophys. 214, 47.

F. KRAUSE: It is worth remarking that in your table with magnetic ages the calculation of the growth rates involves a lot of uncertainties: (i) the magnetic field mode is treated by an approximation; (ii) it is based on estimated values of $\alpha$ and (iii) the differential rotation (Oort's constant). So it appears quite doubtful whether you can make reliable differences between different galaxies.

SHUKUROV: Our results are correct at least to the order of magnitude. Comparison with observations confirms this.

F. KRAUSE: In your latest diagram the parameter $\varepsilon$ probably describes a perturbation and must therefore be small, say $\varepsilon * 1$. The value $\varepsilon_{0}$, where the wanted effect appears (growth rate of the BSS field larger than that of the ASS field), is probably large, I think $\varepsilon>1$. Consequently, $\varepsilon$ is beyond the limit of validity of your calculations. Independent of this, I think that for large $\varepsilon$ this consideration makes no sense, since then all symmetry types appear mixed and no basic symmetry (BSS or ASS) can be identified.

SHUKUROV: Of course the weak-perturbation approach applies only to the cases when the perturbed eigenvalue is close to the unperturbed one. In other words, the only condition of applicability of the perturbation theory is weak deviation from unperturbed state (see e.g. Landau and Lifshitz, Quantum Mechanics). However, for $m>1$ modes the eigenvalues are complex and their imaginary parts are considerably larger than the real parts (Krasheninnikova et al., 1989, Astron. Astrophys. 213, 19). 
Thus the perturbation can lead to a large relative correction of the real part of the eigenvalue, i.e. the growth rate of the field, while the relative correction to the modulus of the eigenvalue is still small.

VOLK: Regarding your arguments about a strong polar field component (dipole or quadrupole) in the central regions of galaxies, I want to point out that in these central regions there is often strong activity. This should then lead to a mass outflow which combs out the field in polar directions. Thus it might be important to consider this alternative, if one compares the observations with dynamo theory.

DEINZER: Völk's remark may be especially important, because in the frame of dynamo theory you can only produce magnetic fields whose toroidal and poloidal parts are at most of the same order of magnitude. But much higher field strengths would be necessary in the case of jets coming out of the central region of galaxies.

SHUKUROV: Concerning the ratio of poloidal to toroidal components of magnetic fields produced by dynamos, the $\alpha \omega$ dynamo gives the dominant toroidal component while the $\alpha^{2}$ dynamo produces them at comparable strength. Explanation of dominance of the poloidal field probably would require us to invoke other additional mechanisms like gas outflows mentioned by Völk which can modify the dynamo-generated field.

PUDRITZ: There is a large difference in the star formation rates between galaxies, and hence on the relative proportion of hot phase, scale height, etc. of their ISM. Could you speculate on the effect this might have on the dynamo theory you have discussed?

SHUKUROV: The mean-field generation models depend on averaged parameters of interstellar gas: rotation, velocity, turbulent magnetic diffusivity, the mean helicity. The latter two quantities depend, in turn, on the r.m.s. turbulent velocity which seems to be rather uniform, about $10 \mathrm{~km} \mathrm{~s}^{-1}$ in all galaxies. Variations of the scale height can play a considerable role because galactic dynamos are rather sensitive to the aspect ratio of the disk.

HELOU: How well does dynamo theory work in dwarf irregular galaxies or blue compact dwarf galaxies? And what growth times would you predict for the magnetic field to reach the few $\mu \mathrm{G}$ levels?

SHUKUROV: Dwarf irregular and blue compact dwarf galaxies probably rotate as rigid bodies. Then the dominant dynamo mechanism would be the $\alpha^{2}$ dynamo. Typical growth times are of the order of turbulent diffusion time across a disk or by the factor of a few longer.

KULSRUD: How did you define time $\mathrm{T}$ to grow to observed strength?

SHUKUROV: In order to estimate $\mathrm{T}$, one should know the initial, seed magnetic field. We used the estimate of the random seed field ejected by supernovae and hot stars, $B_{0} \sim 10^{-9} \mathrm{G}$ (see Ruzmaikin et al., 1988, 
Nature 336, 341-346).

KULSRUD: Has anyone derived an equation for $(\delta B)^{2}$ as this may be important in limiting the mean field generated?

SHUKUROV: The problem of nonlinear dynamo is far from being complete. We can suggest that the steady-state strength of the magnetic field in $\alpha$ dynamos is determined by suppression of the mean helicity of turbulence. The fluctuation magnetic field, $(\delta \mathrm{B})^{2}$, probably is mirror-symmetric and can affect only $\left\langle v^{2}\right\rangle$ but not $\langle v \cdot \nabla \times v\rangle$, with $v$ the turbulent velocity. We should emphasize that equations for $(\delta B)^{2}$ have been obtained and solved for both mean-field and fluctuation dynamos (see e.g. Ruzmaikin, Shukurov and Sokoloff, 1988, Magnetic Fields of Galaxies, Kluwer).

SOKOLOFF: In galaxies the excitation of mean magnetic field is relatively weak. So to construct a nonlinear dynamo theory we need only to develop a simple perturbation theory, considering nonlinearity as a perturbation.

DOLGINOV: I agree with you that a small nonlinearity may be considered as a perturbation in the theory. But the theory doesn't give yet the field magnitudes of the Sun, planets, etc. So one can't say that the nonlinear dynamo theory exists for these objects.

KUNDT: Dynamo equations are not rigorous. A rigorous property is the vanishing magnetic flux across any surface intersecting an integral number of flux loops. I find it difficult to build up a (globally ordered) bisymmetric structure solely from local injections (and the known motions of the galactic gas). 